\title{
Experimental analyses of metal-composite bonded joints: damage identification
}

\author{
Ricardo de Medeiros, Emanuel Nunes Borges and Volnei Tita*
}

\author{
* Correspondence: \\ voltita@sc.usp.br \\ Department of Aeronautical \\ Engineering, São Carlos School of \\ Engineering, University of São \\ Paulo, Av. João Dagnone, 1100, \\ 13573-12 São Carlos, SP, Brazil
}

\begin{abstract}
The advent of composite co-cured and co-bonded integrated construction in aircraft structures has lead to the replacement of fastened joints with bonded joints between the skins and the stiffeners. Skin-stiffener debondings could occur due to impact or other operational reasons and it is usually internal failure. Damage identification of bonded components, which are often vital elements in many structures, is crucial for the prevention of failure of the entire structure. Thus, different researchers have investigated vibration-based methods as an alternative technique to be used in the structural health monitoring (SHM) systems. Hence, this work consists of investigating experimentally through the vibration-based method, the dynamic behavior changes in a bonded metal-composite structure by using piezoelectric transducer and accelerometers in order to monitory the damage. The damage is an artificial debonding in the joint, which was simulated by inserting Teflon ${ }^{\mathrm{TM}}$ tapes within the joint. In-situ inspection as ensured by accelerometer and piezoelectric transducers (PZT) bonded to the structure. Indeed, with a simple comparison of the frequency response functions is difficult to conclude if there is damage in the structure, unless a large damage is presented. However, by using damage metrics, it is possible to identify the damage with more accuracy. Thus, the experimental results obtained by the accelerometers were compared to the data provided by the smart composite sensors (PZT). Finally, it was discussed the advantages and limitations of the experimental analyses and the identification technique proposal.
\end{abstract}

Keywords: Bonded joints; Smart composites; Experimental dynamics analyses; Structural health monitoring

\section{Background}

During the last decades, mainly driven by the aircraft industry, shipbuilding and power generation, numerous studies have been conducted in order to establish the use of composite materials as an alternative safe, effective and economically viable in the development of new products. In the case of reinforced polymeric composites, it is possible to perform assembly by a bonding process between both composite-composite and metal-composite parts. According to Higgins (2000), adhesive bonding of aeronautics primary structures is intensively used on current aircraft projects as a direct alternative of riveting process. However, the degradation of the adhesive layer over the time remains an issue and the inspection of the adhesive layer is complex task since subsurface damages must be removed. Traditional non-destructive techniques (NDT) utilize a variety of methods, ranging from a simple tap test to more complicated approaches, like ultrasonic or thermography techniques. Each of these techniques is 
limited in accuracy and applicability. Also, a significant amount of equipment and expertise is required to perform the inspection procedures. Although there is some limited success in specific cases, in general, NDT methods have proven successful for bond-line assessment (Qing et al. 2006a).

To overcome these limitations (accuracy and application), it is necessary to develop a cost-effective in-service Structural Health Monitoring (SHM) system to monitor and to assess the instantaneous state of aircraft structures. Thus, several SHM approaches have been developed and evaluated for monitoring bonded joints. Chiu et al. (2000) developed a "perceptive repair" or smart system, which will provide information about the in-service performance of the repair and the associated structure. The focus was based on the identification of debonding in the adhesive layer between the repair and the metallic parent part. Also, the authors investigated some criteria related to smart system, such as economic, reliable and self-powered. Finally, the authors proposed that a piezoceramic material could be used in the smart system, because of easy application. Zou et al. (2000) presented a review on the model-based delamination identification methods and the application of vibration-based model-dependent damage identification methods in composite structures.

Mickens et al. (2003) developed a simple vibration-based method of damage identification for monitoring ageing structures. The method intended to detect damage during operation of the aircraft before the damage propagation and the catastrophic failure of aircraft components. The technique used four piezoelectric patches alternatively as actuators and sensors in order to send and receive vibration diagnostic signals. The results obtained by the authors aided to concept a sensor tape in order to detect damage in joints of aircraft structures. Baker et al. (2004) developed methodologies for simulating structural health monitoring (SHM) systems considering adhesively bonded composite repairs of Australian military aircraft. In particular, there was an emphasis on the development of techniques for embedding optical fiber sensors to produce SHM systems. Ogisu et al. (2006) presented a feasibility study for employing a damage monitoring system by using a PZT (piezoelectric) actuator and a Fiber Bragg Grating (FBG) optical fiber sensor. The authors showed that it could be detected several types of damage, such as delamination and debonding. Thus, a conceptual design was implemented in order to employ the novel system. Compressive tests were carried out using the coupon specimens with an embedded small-diameter or standard-diameter optical fiber sensor. In addition, it was verified by the researchers that the coupon specimen with an embedded small-diameter optical fiber did not show any degradation of its material properties.

Qing et al. (2006a) introduced a real-time active Smart Patch System (SPS) based on smart layer technology for monitoring the integrity of bonded repairs. Three applications were presented: (1) monitoring of the cure progress of the bonded repair adhesive, (2) identification of the initial artificial debonding between the composite patch and the metal structure, and (3) monitoring of the damage repaired by a bonded patch, which is under fatigue cycles. Qing et al. (2006b) investigated experimentally the effect of adhesive thickness and its elastic modulus on the performance of adhesively bonded piezoelectric elements, which are used for structural health monitoring. The piezoelectric elements were adhesively bonded to aluminum plates. Hence, the experimental results showed that an increase in adhesive thickness changes the electromechanical 
impedance and the resonant frequency of the piezoelectric elements as well as the amplitude of the sensor signal.

White et al. (2007) presented experimental investigations on representative carbonepoxy composite scarf and over-ply joints. Piezoelectric elements were used to excite and measure the response of the repaired structure. The frequency response signature of the repaired structure with simulated debondings was found to differ significantly from that one with undamaged repair, considering two sets of boundary conditions. Soejima et al. (2008) developed a novel damage monitoring system, which can monitor the integrity of composite structures in aircrafts. In that system, FBG sensors were used as sensors, and piezoelectric transducers (PZT) were used as the generators of elastic waves, which propagated in the structure to be inspected. Damages such as debonding and delamination were introduced in the bonded sections of the skin and stringers by impact loadings. Zagrai et al. (2008) proposed a structural health monitoring (SHM) approach based on nonlinear ultrasonic response for rapid diagnostics of structural connectors and joints. Experimental studies showed variation in the nonlinear response of the joints due to the applied loadings.

Baker et al. (2009) demonstrated the effectiveness of the strain-based SHM approach for monitoring the boron-epoxy patch repair of a critical fatigue crack in F-111C wing. In addition, conventional strain gages were used in the SHM system. White et al. (2009) described a development of a SHM technique for the identification of debonding in composite bonded patches based on frequency responses. Two commonly used repair schemes, the external doubler repair and the scarf repair, were investigated by the authors. Experimental analyses were performed by using the frequency responses of the repairs with and without defects, considering different boundary conditions. It was verified that damage could be detected through changes in the frequency responses for both types of repairs. Quaegebeur et al. (2011) proposed a structural health monitoring strategy in order to detect debondings in a composite lap-joint. The investigated structure was a composite carbon-epoxy part bonded to a titanium plate, and artificial debondings were simulated by inserting Teflon ${ }^{\mathrm{Tm}}$ tapes of various dimensions within the joint. Finite element analyses and experimental tests were carried out in order to validate the efficient identification of the damage and to evaluate the accuracy of damage size estimation. Esmaeel et al. (2011) calculated the Energy Damage Index (EDI) based on a novel vibration-based damage identification methodology by using the Empirical Mode Decomposition (EMD), which is used to predict damage due to absence of bolts in common industrial bolted joints. Finite element model, which use the implicit dynamic solver of the commercial software $\mathrm{Abaqus}^{\mathrm{T \omega}}$, and experimental tests were evaluated. Results showed that the EDI based on the EMD method is a powerful tool not only for detecting the damage, but also for estimating the progression of the damage in bolted joints.

Medeiros et al. (2012) presented a case study about the usage of health monitoring metrics and techniques for detecting damage by using numerical simulations (Finite Element Analysis) and experimental data (vibration test) of a cantilever beam. The approach based on Frequency Response Function (FRF) is used. Also, Medeiros et al. (2013) presented an investigation about the damage effects on the structural response, considering filament winding composite tubes damaged by impact loading. The computational analyses were carried out by using an impulse load, 
which excited the structure, and a piezoelectric transducer, which measured the output data. The results showed that the application of vibration-based methods for detecting damage is feasible. Borges (2012) investigated experimentally and numerically via the vibration-based method the changes in a metal-composite bonded joint by using piezoelectric patch and accelerometers in order to monitor the damage in the joint. That damage, artificial debonding, was simulated by inserting Teflon ${ }^{\mathrm{Tm}}$ tapes within the joint.

As observed at the literature discussed earlier, it is possible to verify many works about structural health monitoring in bonded joints, but it is not common to find scientific contributions, which study metal-composite bonded joints. Moreover, considering these specific contributions, it is very rarely to have works about structural health monitoring of Carbon Fiber Reinforced Polymer (CFRP) parts bonded to a titanium plate with or not damage by using vibration-based method, as well as, by piezoelectric transducer and accelerometers to measure the frequency response function. The design of a SHM system involves the integration, acquisition, analysis, visualization and interpretation of data. The data acquisition system should eliminate aliasing and characterize the signal of interest, while minimizing the amount of data processing. In fact, the study of data features required to distinguish the damaged structures from undamaged ones has received considerable attention in the technical literature. Any SHM monitoring system will produce an enormous amount of data from which it will be necessary to select the appropriate information. An innovative analysis of measured data and accurate interpretation of extracted features are required due to the enormous amounts of data collected during monitoring exercise, which can provide an effective diagnostic and/or prognostic of the structure. Thus, a successful SHM system involves selection and placement of sensors suitable for measurement of key parameters, which influence the performance and the integrity of the structure. Therefore, this work consists on investigating experimentally through the vibration-based method, the dynamic behavior changes in a bonded metal-composite structure by using piezoelectric transducer and accelerometers in order to monitoring a damage. In this work, the damage is an artificial debonding in the joint, which was simulated by inserting Teflon ${ }^{\mathrm{Tx}}$ tapes within the joint. In-situ inspection was ensured by accelerometer and piezoelectric transducers (PZT) bonded to the structure. Indeed, analyzing of FRFs (frequency response functions) and/or mode shapes, obtained via the traditional Fourier Transform (FT), it was possible to detect failure. Thus, the experimental results obtained from the accelerometers were compared to the experimental results provided by the smart composite sensors (PZT). Finally, it was discussed the advantages and limitations of the experimental analyses and the identification technique, which depends on the vibration-based methods.

\section{Methods: specimens manufacturing and experimental analyses}

In the present study, single lap metal-composite bonded joints of titanium and CFRP parts were manufactured by using an epoxy adhesive film. The CFRP plate is made of 7 plies stacked in $\left[0 / 90^{\circ}\right]_{7}$ layup configuration. This material is specified by $\mathrm{Hexcel}^{\mathrm{mm}}$ as M20/G0904/47 (epoxy and carbon fiber bidirectional), which is an epoxy resin M20 reinforced by bidirectional textile carbon fiber G0904. After the cure process recommended by Hexcel ${ }^{\mathrm{Tx}}$, the CFRP plate has $47 \%$ fiber volumetric fraction. In fact, the 
composite parts are obtained from a composite plate following the specifications provided by Military Handbook (2002) and by Hexcel ${ }^{\mathrm{m}}$, using a vacuum bag method and hand layup lamination process. This manufacturing procedure was selected, because it is the most widespread and it is used in aeronautic repair situations, involving such materials. It is important to mention that the vacuum bag technique ensures versatility and operational facility, which can be performed under controlled environment (e.g. laboratories) or even in field (for instance, a repair installed in the aircraft component). In addition, thermal blankets have been used to promote the heating of the composite material during the curing process (c.f., Table 1). Subsequent to this process, the composite plate was cut into six specimens with $390 \mathrm{~mm}$ of length, $25.5 \mathrm{~mm}$ of width and $1.7 \mathrm{~mm}$ of thickness. It is noteworthy that the use of seven layers is due to the fact that many different structures with thicknesses ranging from 1.5 to $2.0 \mathrm{~mm}$ were normally found in the aircraft maintenance area.

The metallic part was made of a titanium alpha-beta alloy Ti6Al4V (AMS4911). This material has excellent mechanical properties as well as impact resistance. Thus, in order to cut the titanium parts from the plates, it was used a guillotine-type knife parallel. Then, it was made up milling the edges for better finishing and assembly of the final set. The titanium part geometries consist on $390 \mathrm{~mm}$ of length, of $25.9 \mathrm{~mm}$ of width and $1.6 \mathrm{~mm}$ of thickness.

The adhesive is a film EA934NA epoxy made from Henkel ${ }^{\mathrm{pw}}$. However, Hysol EA934NA is a bi-component thixotropic paste adhesive, which cures at room temperature and possesses better strength values when the cure process occurs at $300^{\circ}$ $\mathrm{F}\left(149^{\circ} \mathrm{C}\right)$. The thixotropic nature and good compressive strength values are very important for potting, filling and fairing, as well as for shim applications. Hysol EA934NA is qualified as MMM-A-132 Type 1, Class 3 for room temperature cure. It is important to highlight that the damage is an artificial debonding in the joint, which was simulated by inserting Teflon ${ }^{\text {Tx }}$ tapes within the joint.

In order to carry out the vibration tests of the metal-composite bonded structures, it was used an accelerometer and a piezoelectric transducer (smart composite). By one side, the PCB Piezotronix accelerometer (part number 333B30) has the following specifications: modal array, ceramic shear ICP, sensitivity $100 \mathrm{mV} / \mathrm{g}$, measurement range from 0.5 to $3 \mathrm{k} \mathrm{Hz}$. By other side, the smart transducer is made of Lead-ZirkonateTitanate (PZT) ceramic type Midé QP10n. In fact, the piezoelectric transducer consists of a piezoelectric layer made of PZT ceramic and epoxy matrix, which is covered with thin electrodes on the top and bottom side. More details about this transducer can be found at Medeiros (2012).

Table 1 Cure cycle for the specimen (ASTM D3039/D3039M)

\begin{tabular}{cc}
\hline Parameters & Values \\
\hline Vacuum (in. Hg) & 23 \\
Heat up $\left({ }^{\circ} \mathrm{C} / \mathrm{min}.\right)$ & 3 \\
Cure temperature $\left({ }^{\circ} \mathrm{C}\right)$ & 120 \\
Time dwell $(\mathrm{min})$. & 90 \\
Cool down $\left({ }^{\circ} \mathrm{C} / \mathrm{min}.\right)$ & -3 \\
Final cure temperature $\left({ }^{\circ} \mathrm{C}\right)$ & 50 \\
\hline
\end{tabular}


Piezoelectric ceramic is capable of providing a very precise signal of voltage due to very small amounts of strain, which is required for sensor application. The same effect is true in reverse, i.e. a signal of strain is provided by the transducer due to the voltage applied. Hence, a controlled input signal can produce an efficient response in the material, when the device is used as an actuator. In this work, the PZT transducer was used only as a sensor, not as an actuator. The piezoelectric transducer was bonded in the titanium surface by using a vacuum bag for compacting and removing volatile during the cure process of the adhesive, which was performed at room temperature. Figure 1 shows the entities used during the experimental tests and important specifications of dimensions.

Four experimental models were studied in order to represent different scenarios, which the joint can be subjected in operation (in service - Figure 2). The first model (Model 1) represents the intact joint, i.e. without damage. It should be used as reference for comparison with other cases. Therefore, the dynamic responses for the undamaged structure were treated as the dynamic signatures of the joint. The second model (Model 2) contains a damage area in the joint, which corresponds to $50 \%$ of debonding. As commented earlier, these two first models were monitored by accelerometers in order to identify the damage influence on the dynamic behavior of the joint. After that, the third and fourth models (Model 3 and Model 4) were similar to the first e second ones, respectively. However, these models were monitored by PZT transducer attached to the metallic plate (c.f., Figure 2). Thus, the models can evaluate the monitoring capacity of the metal-composite bonded joint, undamaged and damaged, by accelerometer and piezoelectric transducers. As commented previously, in order to simulate the debonding, a constant non-adhering film (Teflon ${ }^{\mathrm{Tm}}$ film) is placed between the adherents (metallic and composite parts) during the curing process of the metal-composite bonded joint.

The experimental analyses consist of verifying dynamically the response of the metalcomposite bonded joints. The data acquisition set-up used in the tests was controlled by a PHO 200 LDS (signal acquisition Photon II), which is a plug and play, multifunction analog, digital and timing I/O board for USB bus computers (Figure 3). The input signals were generated by using an impact force hammer (PCB Piezotronix part number: 0860 - Figure 3). This type of input can excite over a wide range of frequencies. This is important, because different damages can affect different frequency ranges of a

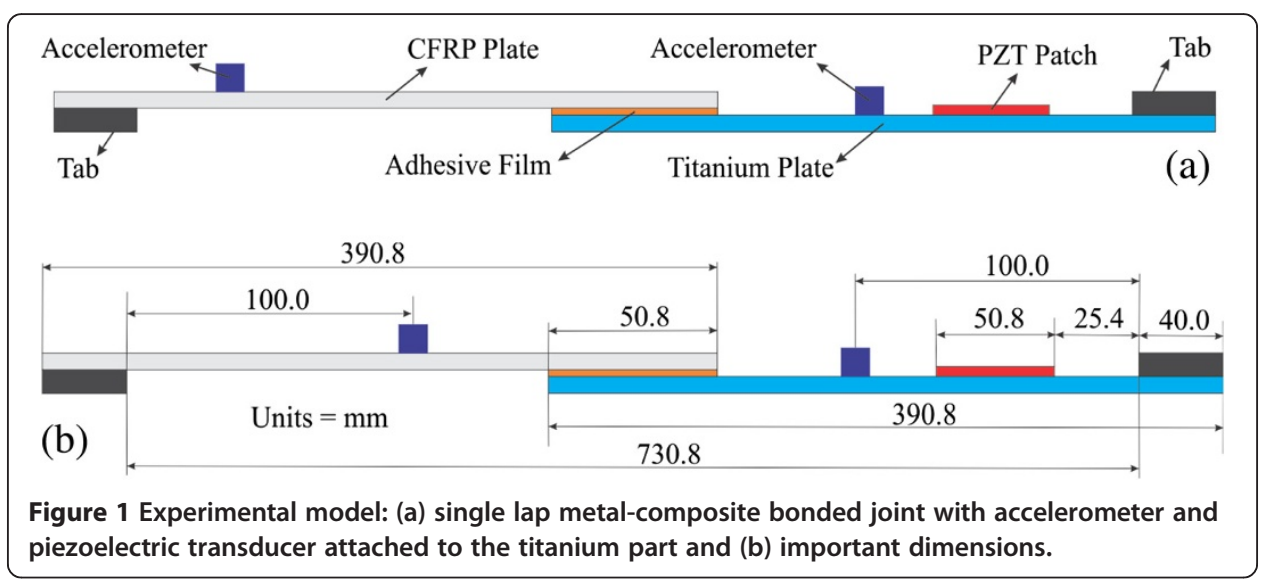




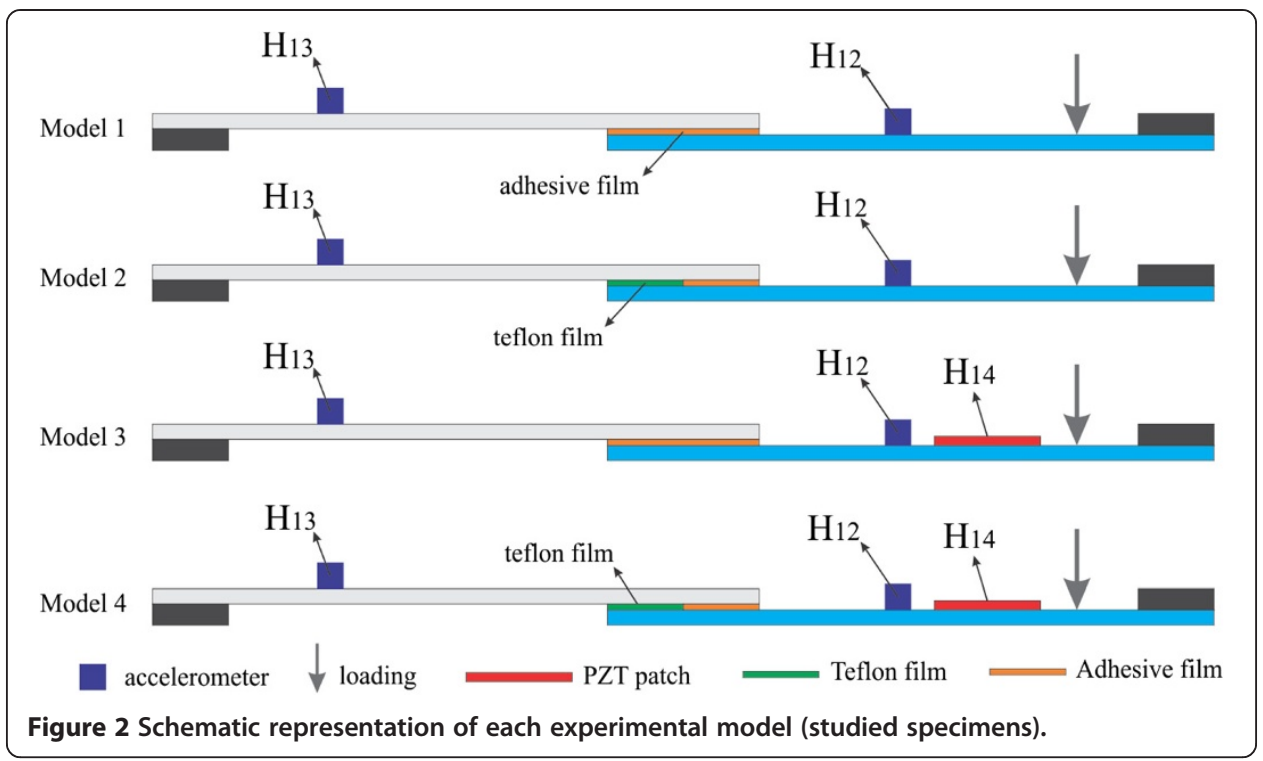

structure, and the resonant and antiresonant characteristics of a structure are good indicators of damage. In fact, this approach is a more global indicator of damage compared to other methods, which use single frequency tone bursts and wave reflection. The FRFs can indicate damage, which is inside the structure, whereas they may not be as sensitive to small damage on the surface as compared to wave propagation methods.

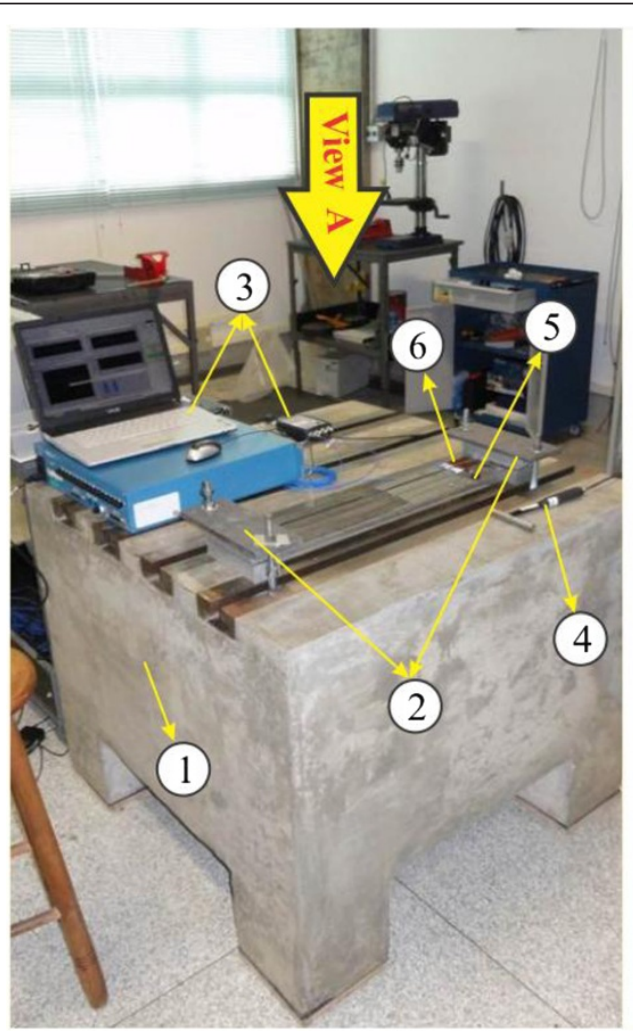

\section{1 - Inertial base}

\section{2 - Both end}

Campled

3 - Photon II PHO 200 LDS

\section{4 - Impulse Hammer PCB Piezotronix Number 0860}

\section{5 - Accelerometer PCB Piezotronix Number 333B30}

\section{6 - PZT Pacth Midé QP10n}


Some important aspects need to be considered due to the specimen assembly. For this reason, all the specimens were assembled together in order to minimize undesired effects caused by the clamped devices, for example, pre-stress due to assembly (Figure 4). However, these effects were not evaluated during the experimental analyses, because the specimens were fixed together and the axial load created by the clamped device was kept as low as possible in order to avoid changes in the dynamic behavior of the specimens (c.f., Figure 4). However, it was not considered the vibration effects from one specimen to others and vice-versa.

Based on the experimental set-up shown by the Figure 4, it was investigated the vibration-based damage identification for metal-composite bonded joints. It is important to notice that the fundamental idea for this method consists on the principle that the damage changes the physical properties (damping and stiffness) of the structures. Hence, these changes cause modifications in modal properties (natural frequencies, modal damping and mode shapes). For instance, it is possible to observe reductions in stiffness due to cracks. Therefore, damage can be identified by analyzing the changes in vibration behavior of the structure. Hence, the knowledge of the vibrational behavior of a structure can be used to determine the existence as well as the location and extension of damage.

As well known, dynamic responses can be expressed in the time or frequency domain. For linear systems, there is a little loss of information when the data are converted from the time domain to the frequency domain. In this work, the FRFs were obtained from the ratio between the FFT (Fast Fourier Transform) of the response (output) and the FFT of the excitation (input). Thus, an impulse force signal was used to excite the structure (input) and the output was measure by using PZT transducer and accelerometers. In each case, excitation signals from an impact hammer were applied as perpendicular loading on the titanium part, and the output signals were obtained from the positions, where accelerometers $\left(\mathrm{H}_{12}\right.$ and $\left.\mathrm{H}_{13}\right)$ and PZT sensor $\left(\mathrm{H}_{14}\right)$ were attached (c.f., Figure 2). Each time signal gathered consisted of 8192 points and

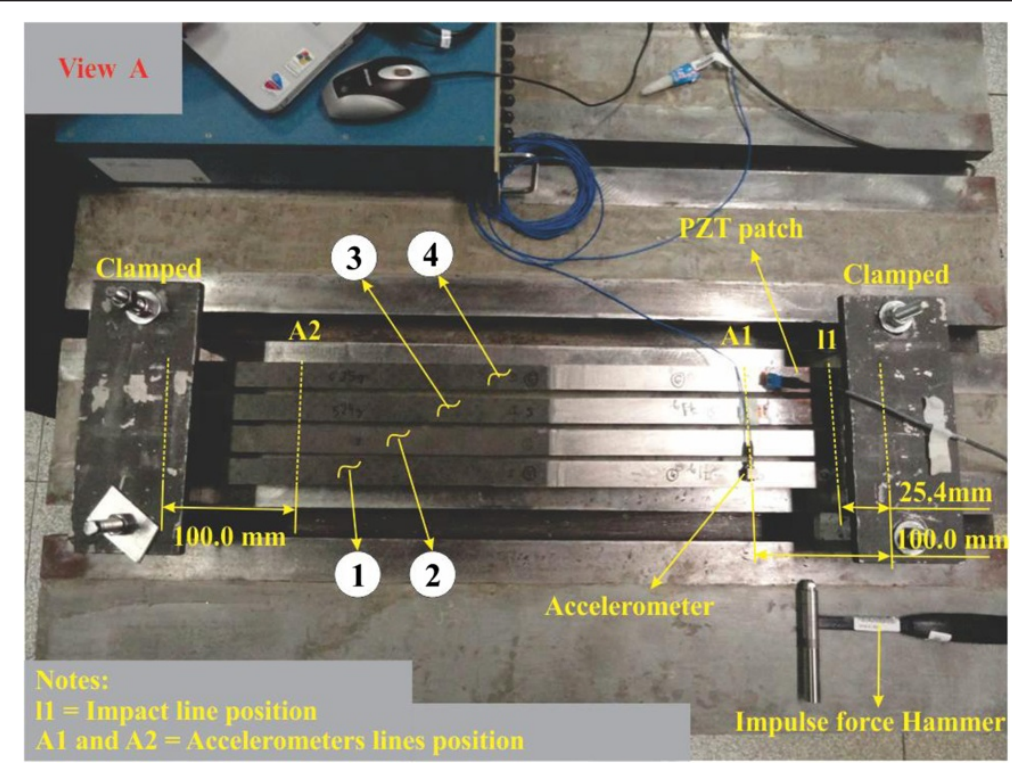

Figure 4 Assembly and position of the specimens. 
they were sampled until $1000 \mathrm{~Hz}$. The number of averaging individual time records was selected to be five in order to reduce the random fluctuation in the estimation of the FRFs. After the data acquisition, they were saved in a file to be analyzed by using a signal processing software (Dynamic Signal Analyzer - Photon II). Finally, in order to identify the debonding damage, the FRFs for undamaged and damaged joints were compared.

\section{Results and discussion}

First of all, the peaks of the measured FRFs contain significant information of the metal-composite bonded joints, such as natural frequencies, damping and, it may be possible to identify damage. In order to evaluate the potentialities and limitations of SHM system, which uses vibration-based damage identification, three case studies were investigated by using the experimental models shown previously. In the first case study (Case Study 1), it was not only investigated the vibration feature of the metalcomposite bonded joint, but also the influence of the PZT transducer in the dynamic structural response. Hence, in this study, it was used the Model 1 and the Model 3. In the second case study (Case Study 2), it was evaluated the damage model by using accelerometer (without PZT transducer). Thus, it was used the Model 1 and the Model 2. Finally, the third case study (Case Study 3) consists of evaluating the damage model by using PZT transducer. Therefore, it was analyzed the Model 3 and the Model 4. It is worth to mention that once the frequency increases with the presence of PZT transducer, the FRF response becomes more dependent on the structure in the neighborhood of the sensors.

\section{Case study 1 - influence of PZT transducer}

The experimental Model 1 (intact without PZT sensor) was carried out in order to use as a reference for the development of the vibration characteristic for the metalcomposite bonded joint. The experimental Model 3 (intact with PZT sensor) was performed in order to verify the influence of the piezoelectric sensor in the dynamic structural response. Thus, there is a comparison between the experimental FRFs, measured by accelerometers, for the both end clamped joints with and without piezoelectric transducer (c.f., Figure 5 and Figure 6). Based on the dynamic responses, it is noted primarily the changes of natural frequencies. These changes are due to not only the increase of mass, but also by the changes in the stiffness with the presence of the PZT sensor.

Regarding the signal measured by accelerometer, it is important to observe that the FRF curves are much clearer below $500 \mathrm{~Hz}$. Thus, it may be difficult to observe changes in the FRFs due to debonding, considering frequency ranges higher than $500 \mathrm{~Hz}$. Another important aspect to be observed regarding the accelerometer signal was a slight difference in the signal from the accelerometer $\left(\mathrm{H}_{13}\right)$ with and without the PZT transducer, especially between $200 \mathrm{~Hz}$ and $350 \mathrm{~Hz}$ (c.f., Figure 6).

The Table 2 clearly demonstrated the differences between the data obtained from the experimental analyses for the Model 1 and Model 3. The largest difference is for the $2^{\text {nd }}$ mode obtained by $\mathrm{H}_{12}$ and for the $4^{\text {th }}$ mode by $\mathrm{H}_{13}$, which is less than $4 \%$. This difference can be explained by the accelerometers positions. 

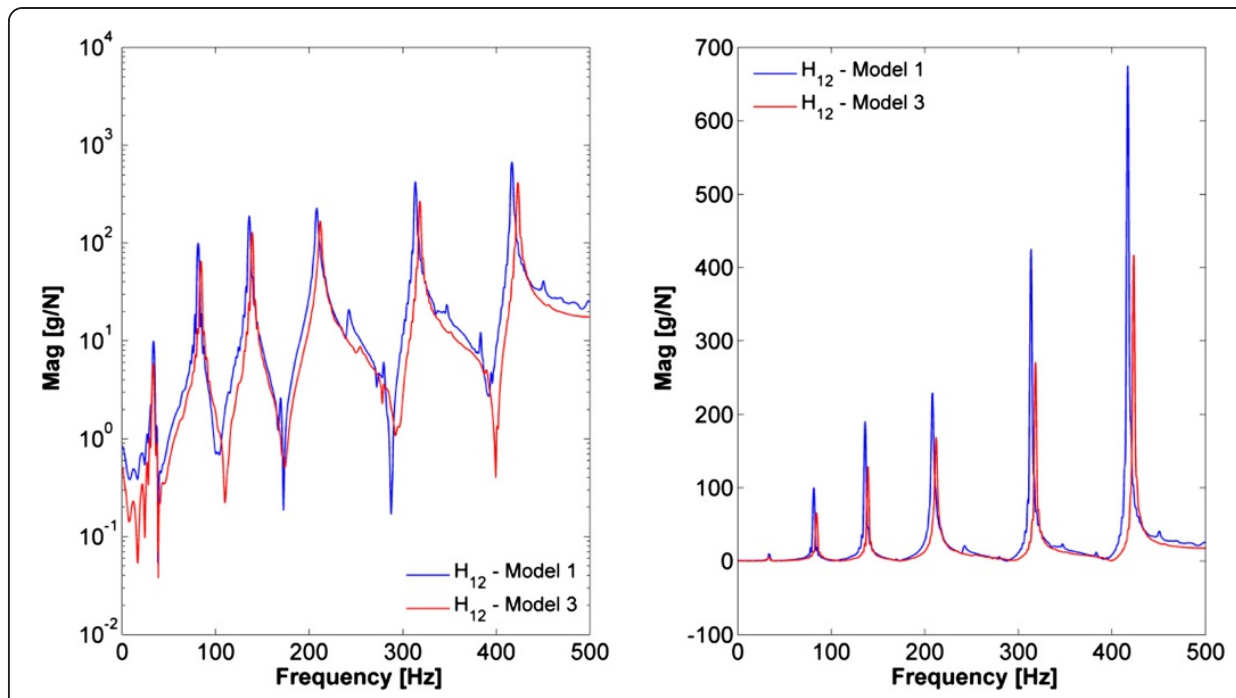

Figure 5 With vs. without piezoelectric transducer FRFs for $\mathrm{H}_{12}$, Model 1 and Model 3 - Case Study 1.

\section{Case study 2 - damage identification without PZT transducer}

The case study 2 consists of analyzing metal-composite single lap joint specimen, with and without damage, by using accelerometers. The FRFs for the intact and damaged joint are shown in Figure 7 and Figure 8. Regarding the damage in the experimental Model 2, it is shown that it lost structural stiffness due to the debonding failure. As commented earlier, the experimental tests were carried out by using the same set-up for both models, i.e. it was used the same assembly. Thus, the changes observed in the FRFs could not be created by the differences in assembly procedures.

The Table 3 demonstrated the differences between the data obtained from the experimental analyses for the Model 1 and Model 2. The largest difference is for the $5^{\text {th }}$ mode obtained by $\mathrm{H}_{12}$ and for the $3^{\text {rd }}$ mode by $\mathrm{H}_{13}$, which is less than $7 \%$. This difference can be explained by the accelerometers positions and the modal shape of the structure.
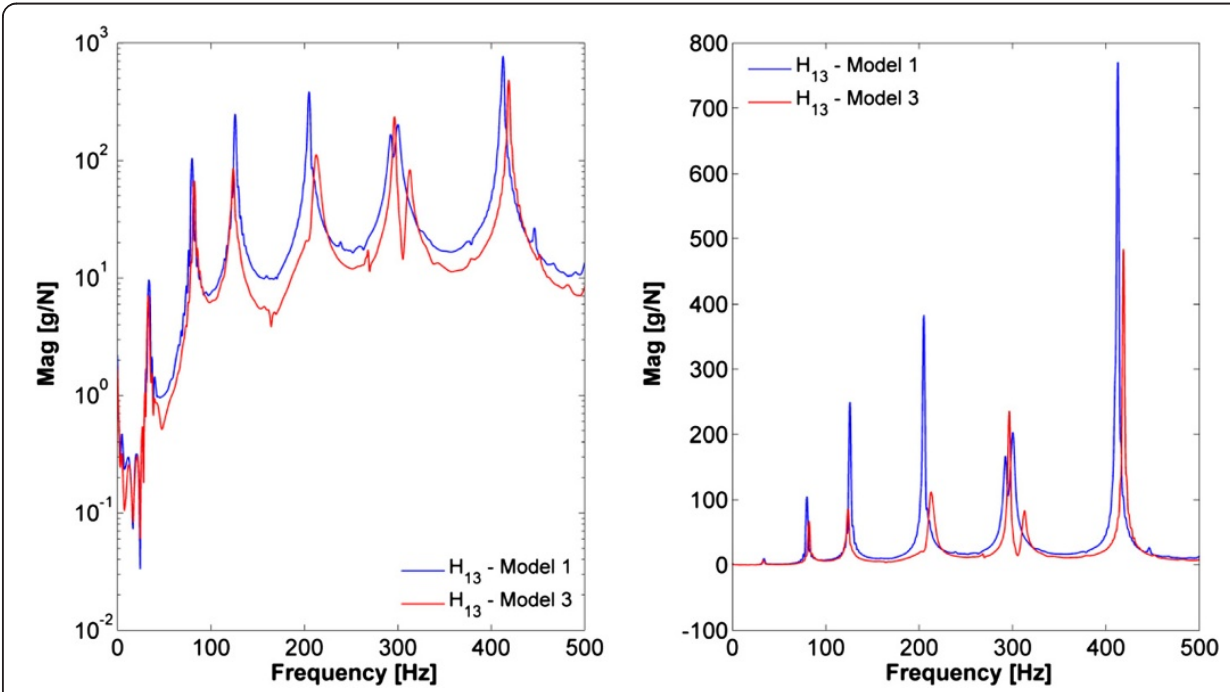

Figure 6 With vs. without piezoelectric transducer FRFs for $\mathrm{H}_{13}$, Model 1 and Model 3 - Case Study 1. 
Table 2 Resonance frequencies obtained by experimental tests for Model 1 and Model 3

\begin{tabular}{|c|c|c|c|c|c|c|c|}
\hline & Mode & $\omega_{1}[\mathrm{~Hz}]$ & $\omega_{2}[\mathrm{~Hz}]$ & $\omega_{3}[\mathrm{~Hz}]$ & $\omega_{4}[\mathrm{~Hz}]$ & $\omega_{5}[\mathrm{~Hz}]$ & $\omega_{6}[\mathrm{~Hz}]$ \\
\hline \multirow[t]{2}{*}{ Model 1} & $\mathrm{H}_{12}$ & 33.44 & 81.25 & 135.9 & 208.1 & 313.4 & 416.9 \\
\hline & $\mathrm{H}_{13}$ & 33.75 & 79.69 & 125.9 & 205.0 & 292.2 & 412.5 \\
\hline \multirow[t]{2}{*}{ Model 3} & $\mathrm{H}_{12}$ & 33.13 & 84.38 & 139.1 & 211.9 & 318.1 & 423.1 \\
\hline & $\mathrm{H}_{13}$ & 33.13 & 81.88 & 124.1 & 212.5 & 296.3 & 418.8 \\
\hline \multicolumn{2}{|c|}{$\Delta^{(*)}: H_{12}$} & $0.93 \%$ & $3.85 \%$ & $2.35 \%$ & $1.83 \%$ & $1.50 \%$ & $1.49 \%$ \\
\hline \multicolumn{2}{|c|}{$\Delta^{(*)}: \mathrm{H}_{13}$} & $1.84 \%$ & $2.75 \%$ & $1.43 \%$ & $3.66 \%$ & $1.40 \%$ & $1.53 \%$ \\
\hline
\end{tabular}

${ }^{*} \Delta=|H(M 1)-H(M 3)| / H(M 1)$

\section{Case study 3 - damage identification with PZT transducer}

The case study 3 was carried out in order to evaluate the applicability of the vibrationbased monitoring technique by using PZT sensors. For both measured procedures (accelerometer and PZT), it is evident that the damage investigated in this study produces modifications in the FRFs. These changes are shown by the lower frequencies, but they are more pronounced at higher ones (above $500 \mathrm{~Hz}$ ). However, as commented before, the signal response is not so clear for these frequency ranges. In fact, there is a frequency reduction, which can be explained by classical structural dynamics. This behavior was expected, because the joint loses ability to transfer loading with the debonding area in the joint. Comparing the FRFs by using a PZT transducer and the accelerometers, it was verified that the damaged model has lower stiffness than undamaged one (c.f., Figures 9, 10 and 11).

Furthermore, the signals obtained by both the accelerometer and the piezoelectric transducer exhibit good consistency, although there are low differences between the signal from the accelerometer and the PZT transducer (as shown in the Table 4). In addition, the resonance peaks of the PZT are above the peaks presented by accelerometers. This indicates that the PZT transducer also influence on the damping of the joint.
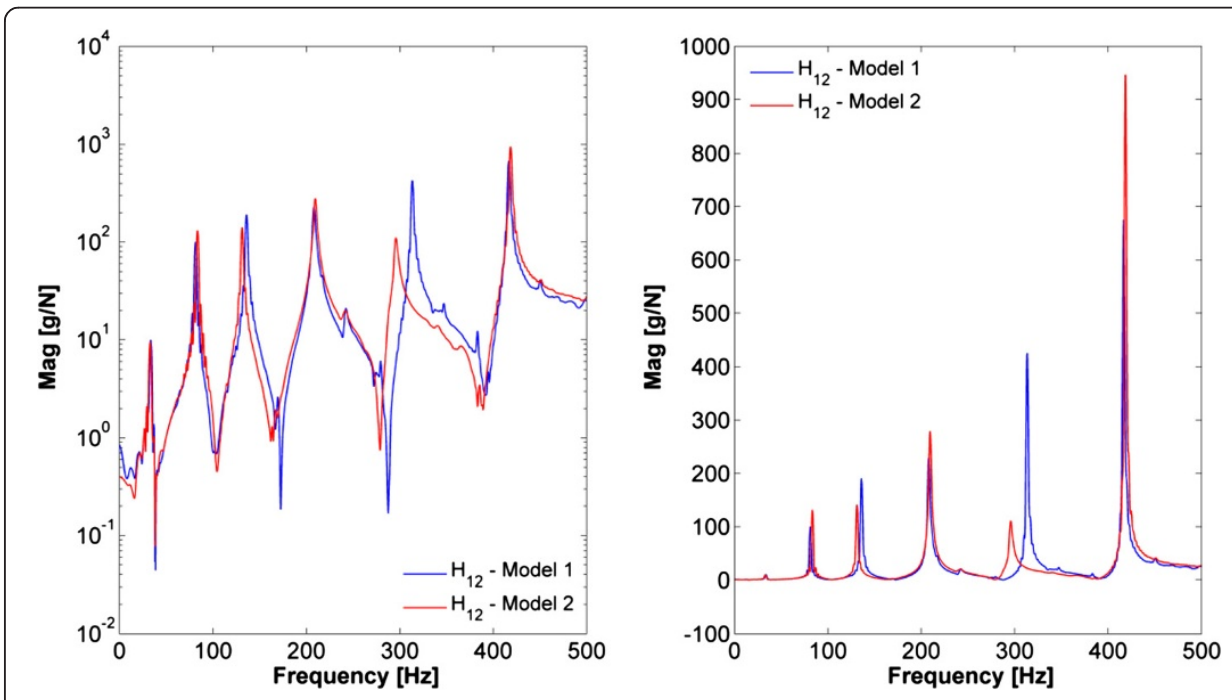

Figure 7 Intact vs. damaged FRFs for $\mathrm{H}_{12}$, Model 1 and Model 2 - Case Study 2. 


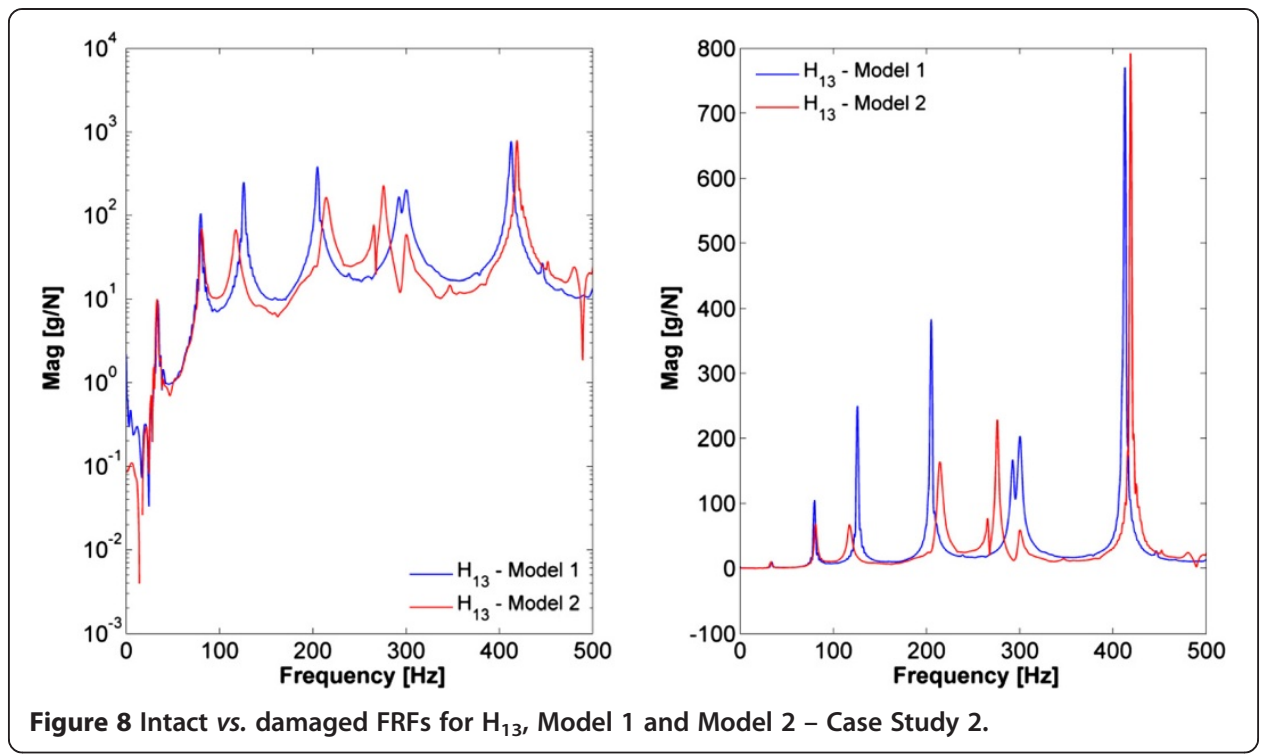

As observed from the FRFs, it is possible to identify the damage in the metalcomposite bonded joint but, sometimes, depends on the size and location of the damage, as well as the interest frequency range, it is very complicated to do this task. By other side, the FRF is desirable from the viewpoint of applications for SHM systems, because structural FRFs are sensitive to small changes and damage in a structure. To quantify this sensitivity, damage indicators have been developed to calculate the difference in the FRF responses between intact (undamaged) and damaged structures.

One very important damage indicator for the structure was developed by Mickens et al. (2003). This damage indicator considers the percent difference between the magnitude of the FRFs of the undamaged and damaged structures. As it is known, any physical quantity can be used to compute the FRF, such as acceleration/force, velocity/ force, displacement/force, strain/force or PZT (piezoelectric) sensor voltage/PZT excitation voltage. Thus, the damage indicator $\mathrm{D}$ can be obtained by computing the mean value of $y(f)$ for the frequency range of interest.

$$
y(f)=a b s\left(\frac{\left|H^{i}\right|-\left|H^{d}\right|}{\left|H^{i}\right|}\right),
$$

Table 3 Resonance frequencies obtained by experimental tests for Model $\mathbf{1}$ and Model $\mathbf{2}$

\begin{tabular}{|c|c|c|c|c|c|c|c|}
\hline & Mode & $\omega_{1}[\mathrm{~Hz}]$ & $\omega_{2}[\mathrm{~Hz}]$ & $\omega_{3}[\mathrm{~Hz}]$ & $\omega_{4}[\mathrm{~Hz}]$ & $\omega_{5}[\mathrm{~Hz}]$ & $\overline{\omega_{6}[\mathrm{~Hz}]}$ \\
\hline \multirow[t]{2}{*}{ Model 1} & $\mathrm{H}_{12}$ & 33.44 & 81.25 & 135.9 & 208.1 & 313.4 & 416.9 \\
\hline & $\mathrm{H}_{13}$ & 33.75 & 79.69 & 125.9 & 205.0 & 292.2 & 412.5 \\
\hline \multirow[t]{2}{*}{ Model 2} & $\mathrm{H}_{12}$ & 33.13 & 83.44 & 131.3 & 209.4 & 295.6 & 418.4 \\
\hline & $\mathrm{H}_{13}$ & 33.13 & 80.94 & 117.2 & 214.4 & 275.6 & 418.8 \\
\hline \multicolumn{2}{|c|}{$\Delta^{(*)}: \mathrm{H}_{12}$} & $0.93 \%$ & $2.70 \%$ & $3.38 \%$ & $0.62 \%$ & $5.68 \%$ & $0.36 \%$ \\
\hline \multicolumn{2}{|c|}{$\Delta^{(*)}: H_{13}$} & $1.84 \%$ & $1.57 \%$ & $6.91 \%$ & $4.59 \%$ & $5.68 \%$ & $1.53 \%$ \\
\hline
\end{tabular}

${ }^{*} \Delta=|H(M 1)-H(M 2)| / H(M 1)$. 

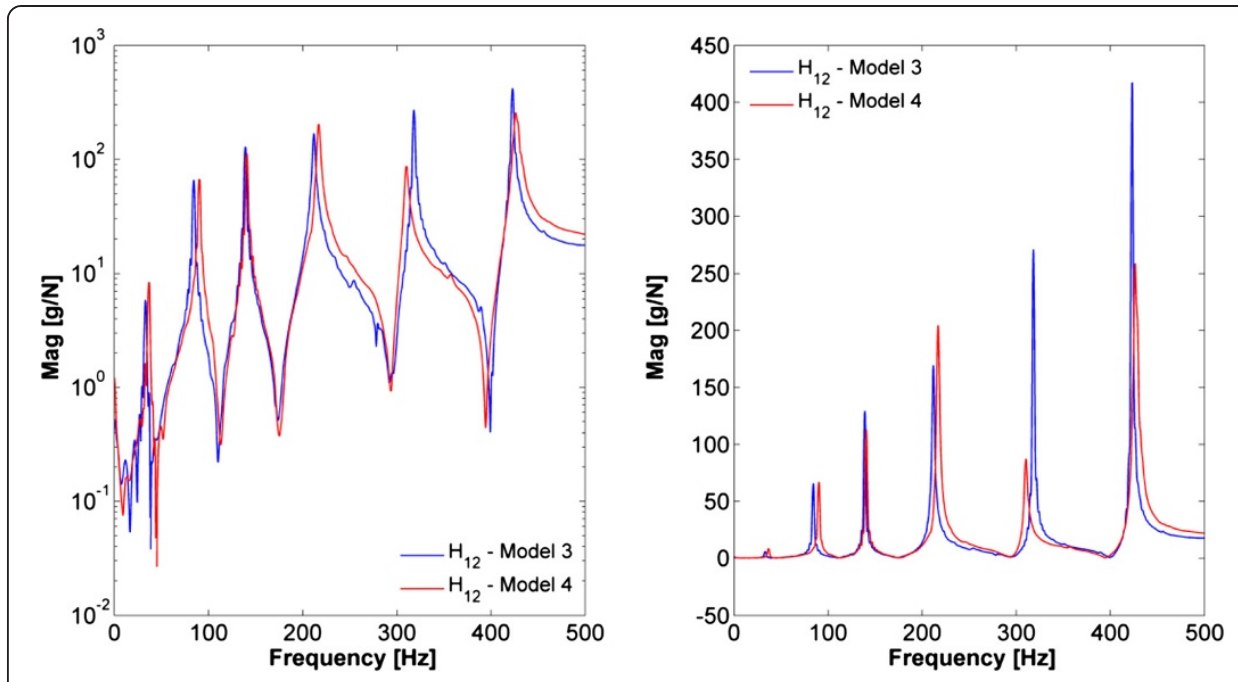

Figure 9 Intact vs. damaged FRFs for $\mathrm{H}_{12}$, Model 3 and Model 4 - Case Study 3.

$$
D=\frac{\Delta f}{f_{2}-f_{1}} \sum_{i}^{n} y_{i}(f),
$$

where the superscripts $i$ and $d$ denote the intact and damage structures, respectively, and the vertical bars represent the magnitude of the function. Also, $f_{1}$ is the lower frequency and $f_{2}$ is the upper frequency of the range of interest and $\Delta f$ is the frequency increment between measurement points. In addition, the equation (2) provides a damage indicator, which gives a normalized measurement of damage in the structure. These values once collected for different sensor/actuator pairs can roughly quantify the amount of damage in a structure. The $D$ expression returns values greater than zero if any variation in the structural dynamic behavior occurs, and D will return "zero", if there is not any damage in the structure.
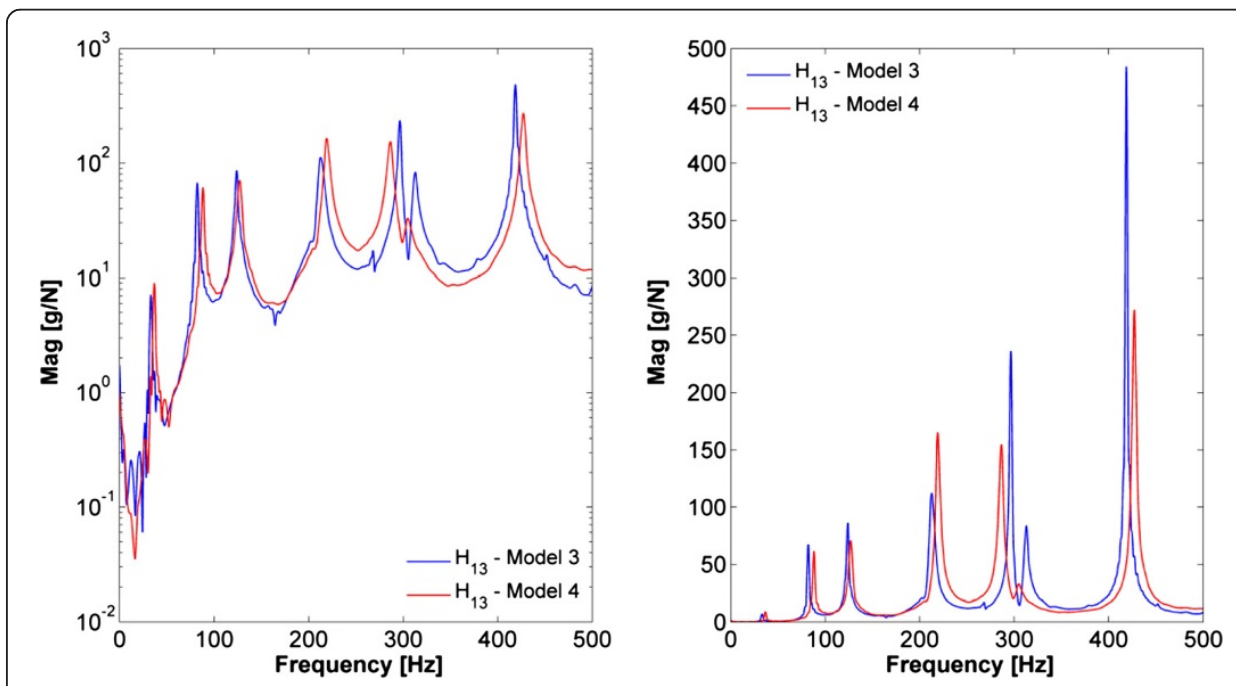

Figure 10 Intact vs. damaged FRFs for $\mathrm{H}_{13}$, Model 3 and Model 4 - Case Study 3. 


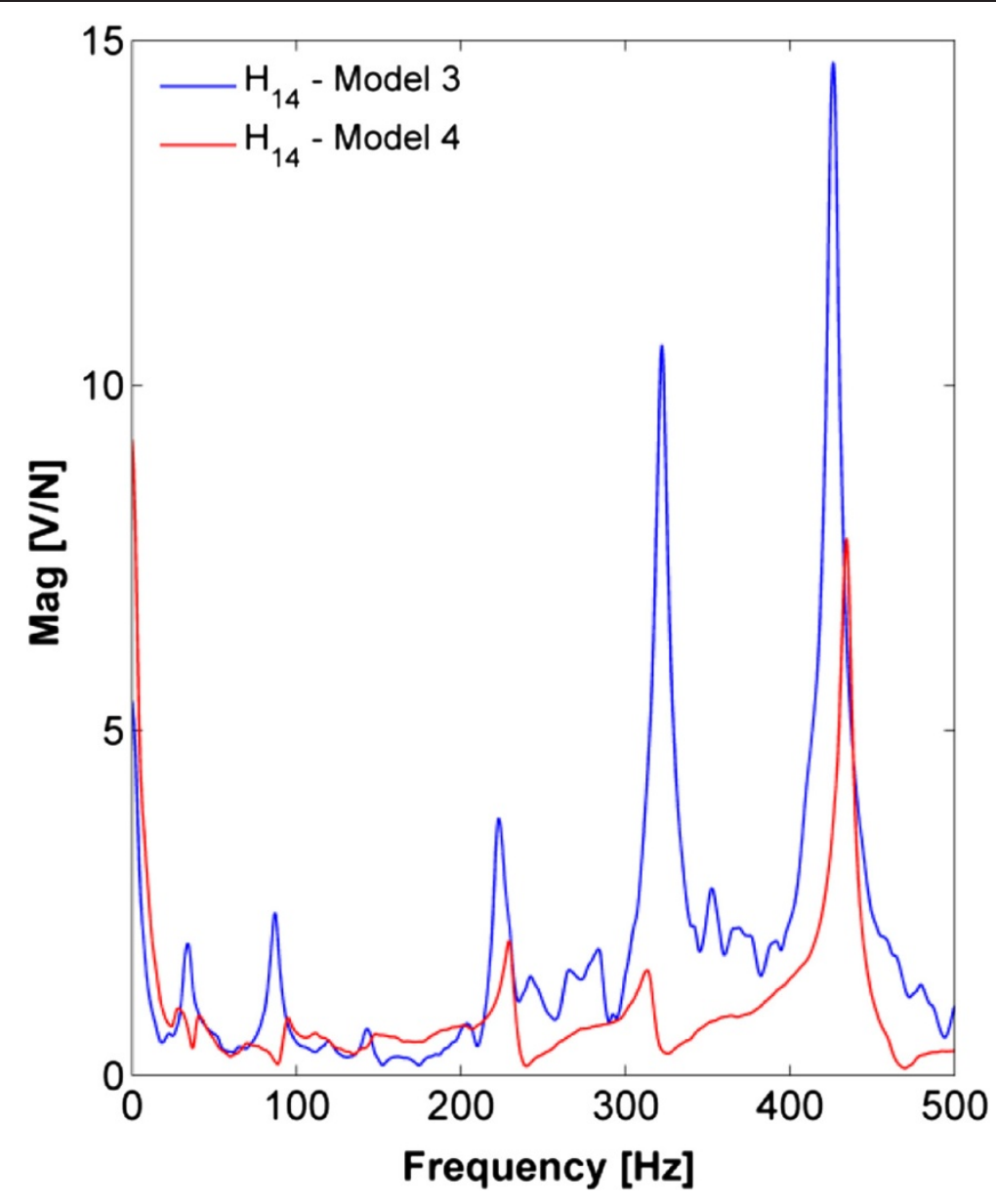

Figure 11 Intact vs. damaged FRFs for $\mathrm{H}_{14}$, Model 3 and Model 4 - Case Study 3.

Other important metric was proposed by Monaco et al. (2000) and uses the changes in measured FRFs in order to determine the Damage Index $(D I)$. This metric is based on the acquisition and comparison of FRFs from the monitored structure before and after damage occurrence. As commented previously, structural damages modify the dynamic behavior of the structure and, consequently, its FRFs. This makes possible the

Table 4 Resonance frequencies obtained by experimental tests for Model $\mathbf{3}$ and Model 4

\begin{tabular}{cccccccc}
\hline & Mode & $\boldsymbol{\omega}_{\mathbf{1}}[\mathrm{Hz}]$ & $\boldsymbol{\omega}_{\mathbf{2}}[\mathrm{Hz}]$ & $\boldsymbol{\omega}_{\mathbf{3}}[\mathrm{Hz}]$ & $\boldsymbol{\omega}_{\mathbf{4}}[\mathrm{Hz}]$ & $\boldsymbol{\omega}_{\mathbf{5}}[\mathrm{Hz}]$ & $\boldsymbol{\omega}_{\mathbf{6}}[\mathrm{Hz}]$ \\
\hline Model $\mathbf{3}$ & $\mathbf{H}_{\mathbf{1 2}}$ & 33.13 & 84.38 & 139.1 & 211.9 & 318.1 & 423.1 \\
& $\mathbf{H}_{\mathbf{1 3}}$ & 33.13 & 81.88 & 124.1 & 212.5 & 296.3 & 418.8 \\
& $\mathbf{H}_{\mathbf{1 4}}$ & 33.75 & 86.88 & 142.5 & 222.8 & 321.9 & 426.3 \\
\multirow{3}{*}{ Model $\mathbf{4}$} & $\mathbf{H}_{\mathbf{1 2}}$ & 36.88 & 90.31 & 140.6 & 216.9 & 310.3 & 426.3 \\
& $\mathbf{H}_{\mathbf{1 3}}$ & 36.88 & 88.13 & 126.9 & 219.1 & 286.6 & 427.2 \\
& $\mathbf{H}_{\mathbf{1 4}}$ & 36.88 & 94.69 & 147.8 & 229.1 & 312.8 & 434.1 \\
$\boldsymbol{\Delta}^{(*)}: \mathbf{H}_{\mathbf{1 2}}$ & & $11.32 \%$ & $7.03 \%$ & $1.08 \%$ & $2.36 \%$ & $2.45 \%$ & $0.76 \%$ \\
$\boldsymbol{\Delta}^{(*)}: \mathbf{H}_{\mathbf{1 3}}$ & & $11.32 \%$ & $7.63 \%$ & $2.26 \%$ & $3.11 \%$ & $3.27 \%$ & $2.01 \%$ \\
$\boldsymbol{\Delta}^{(*)}: \mathbf{H}_{\mathbf{1 4}}$ & $9.27 \%$ & $8.99 \%$ & $3.72 \%$ & $2.83 \%$ & $2.83 \%$ & $1.83 \%$ \\
\hline
\end{tabular}

${ }^{*} \Delta=|H(M 3)-H(M 4)| / H(M 3)$. 
calculation of a representative $D I$. In this approach, the calculated $D I s$ are the averages of the differences between intact and damaged structures. In this work, two DI expressions have been considered:

$$
\begin{gathered}
D I_{1}=\frac{\sum_{j=1}^{n}\left|F I_{i}-F D_{i}\right|}{\sum_{j=1}^{n}\left|F I_{i}\right|}, \\
D I_{2}=\sum_{j=1}^{n} \frac{\left(\frac{\sum_{j=1}^{n}\left|F I_{i}-F D_{i}\right|}{\sum_{j=1}^{n}\left|F I_{i}\right|}\right)}{n},
\end{gathered}
$$

where $F I_{i}$ and $F D_{i}$ are respectively the $\mathrm{n}$ values of the intact and damaged structures FRFs, and $n$ depends on the chosen sampling frequency and frequency bandwidth of acquisition. Both $D I$ expressions return values greater than zero, if any variation in the structural dynamic behavior occurs, and they will return "zero", if there is no damage in the structure.

Another important approach was presented by Prada et al. (2012) and it was defined as Change in Measured Parameters Metric (CMPM). This metric uses previously numerical or experimental modal characteristics of the undamaged structure. After that, it uses the parameters provided by a sensor in the damaged structure. In addition, it has the advantage of being simple to be implemented and only one sensor may be used in the structure.

$$
C M P M=\frac{\left(\frac{\phi}{F_{U D}}\right)}{\left(\frac{\phi}{F_{D j}}\right)_{\text {sensor }}},
$$

where $\phi$ are the resonance frequencies for damage or intact structure. $F_{U D}$ and $F_{D j}$ are the amplitudes for the intact (undamaged) and damaged structure, respectively by the $j$ sensors. This damage index return values close to "one", if there is not any variation in the structural dynamic behavior.

The damage values for these analyses are given in Table 5 and Table 6 . These values were obtained by a piezoelectric $\left(\mathrm{H}_{14}\right)$ and a point, which corresponds to the position of the accelerometer $\left(\mathrm{H}_{12}\right.$ and $\left.\mathrm{H}_{13}\right)$. It is important to notice that $H_{i, j}$ indicates the point, which is loaded $(i)$, and the point, which is the sensor $(j)$, as given by Figure 2. It

Table 5 Damage indicators D and DI for the structure

\begin{tabular}{ccccc}
\hline Damage indicator & Intact & \multicolumn{3}{c}{ Position } \\
\cline { 3 - 5 } & & $\mathbf{H}_{\mathbf{1 2}}$ & $\mathbf{H}_{\mathbf{1 3}}$ & $\mathbf{H}_{\mathbf{1 4}}$ \\
\hline $\mathrm{D}$ & 0.0 & 0.1819 & 0.4949 & 0.1492 \\
$\mathrm{DI}_{1}$ & 0.0 & 0.7731 & 0.6112 & 0.5509 \\
$\mathrm{Dl}_{2}$ & 0.0 & 263.9329 & 97.0329 & 79.5999 \\
\hline
\end{tabular}


Table 6 Damage indicator CMPM for the structure

\begin{tabular}{lccccccc}
\hline Position & Intact & \multicolumn{7}{c}{ Damaged } \\
\cline { 3 - 8 } & & $\boldsymbol{\omega}_{\mathbf{1}}$ & $\boldsymbol{\omega}_{\mathbf{2}}$ & $\boldsymbol{\omega}_{\mathbf{3}}$ & $\boldsymbol{\omega}_{\mathbf{4}}$ & $\boldsymbol{\omega}_{\mathbf{5}}$ & $\boldsymbol{\omega}_{\mathbf{6}}$ \\
\hline $\mathrm{H}_{12}$ & 1.0 & 1.288 & 0.951 & 0.865 & 1.181 & 0.330 & 0.615 \\
$\mathrm{H}_{13}$ & 1.0 & 1.134 & 0.849 & 0.803 & 1.425 & 0.678 & 0.551 \\
$\mathrm{H}_{14}$ & 1.0 & 0.467 & 0.326 & 0.846 & 0.509 & 0.149 & 0.520 \\
\hline
\end{tabular}

was observed that the damage indicator values for $H_{12}, H_{13}$ and $H_{14}$ were different and the highest value was obtained for the accelerometer attached to the composite part. This difference can be explained due to the relation between the sensors and the loading position. As close this distance, as less the interference in the data acquisition.

The seemingly large value of damage indicators occurs, because the damage indicator involves division of FRFs. When damage occurs in the structures with small damping, it is possible to observe that there are misalignments between the FRFs for the intact and damaged structures. Thus, due to many peaks and valleys in the FRFs, small misalignments in the natural frequencies can cause large changes in the calculation of damage indicators functions as shown by the Tables 5 and 6 .

\section{Conclusions}

A damage identification experimental study was shown for single lap metal-composite bonded joint made of titanium and epoxy resin reinforced by carbon fiber. The damage was simulated by using an artificial debonding in the joint, inserting Teflon ${ }^{\text {tw }}$ tapes within the joint. In fact, vibration tests were performed in intact and damaged bonded joints in order to identify the damage via piezoelectric transducer and accelerometers.

The experimental results demonstrated the usefulness of vibration-based method by using accelerometers and piezoelectric transducers (as sensors) in order to detect debonding damage. This method has the advantage to be easily implemented. Also, it can provide the global behavior of the structure as well as local information in real time (e.g. in flight conditions), considering the usage of PZT sensors. Besides, it is very important to highlight that this method do not require direct human accessibility to the structure, and this is very strategic mainly for bonded joints. On the other hand, there are some limitations. Since the method is a difference between the data of the intact and damaged structure, if the data acquisition possesses noise, it is very complicated to identify the damage. This occurs because the comparison between FRFs from intact and damaged can be contaminated mainly in the lower frequencies. Therefore, it is concluded that the identification of the damage cannot be based only on the difference of natural frequencies for intact and damaged structure. Thus, a damage indicator is strongly recommended.

It was shown that the damage metric developed by Mickens et al. (2003) is a good option not only to identify the damage, but also to provide a prediction of the damage severity. However, even using damage metrics, SHM systems based on vibration methods provide little information about the location and extension of the damage, unless large quantities of sensors are employed. And, this will probably increase the cost and the weight of the component.

Finally, regarding the potentialities and limitations shown above, it is possible to conclude that there is a great future perspective for the application of vibration-based methods by using PZT sensors on SHM systems for metal-composite bonded structures. 


\section{Competing interests}

The authors declare that they have no competing interests.

\section{Authors' contributions}

RM - carried out the experimental tests and drafted the manuscript. ENB - carried out the joint studies and participated in the experimental tests. VT - carried out the joint studies, supervised the experimental analyses and corrected the manuscript. All authors read and approved the final manuscript.

\section{Acknowledgements}

The authors are thankful to FAPESP (process numbers: 2012/01047-8), as well as, CNPq (process numbers: 135652/2009-0 and 208137/2012-2) and FAPEMIG for partially funding the present research work through the INCT-EIE.

\section{Received: 29 November 2013 Accepted: 13 December 2013}

Published: 5 May 2014

\section{References}

Baker W, McKenzie I, Jones R (2004) Development of life extension strategies for Australian military aircraft, using structural health monitoring of composite repairs and joints. Compos Struct 66:133-143, doi: 10.1016/j.compstruct.2004.04.031

Baker A, Rajic N, Davis C (2009) Towards a practical structural health monitoring technology for patched cracks in aircraft structure. Compos Appl Sci Manuf 40:1340-1352, doi: 10.1016/j.compositesa.2008.09.015

Borges EN (2012) Manufacturing, computational modeling and experimenting evaluation of hybrid bonded joints monitored through smart composites, Master's thesis. São Carlos School of Engineering, University of São Paulo, São Carlos, São Paulo, Brazil (in portuguese)

Chiu WK, Galea SC, Koss LL, Rajic N (2000) Damage detection in bonded repairs using piezoceramics. Smart Mater Struct 9:466-475, doi:10.1088/0964-1726/9/4/309

Esmaeel RA, Briand J, Taheri F (2011) Computational simulation and experimental verification of a new vibration-based structural health monitoring approach using piezoelectric sensors. Struct Health Monit 11:237-250, doi: 10.1177/1475921711414239

Higgins A (2000) Adhesive bonding of aircraft structures. Int J Adhes Adhes 20:367-376, doi: 10.1016/50143-7496(00)00006-3

Medeiros R (2012) Development of a computational methodology for determining effective coefficients of the smart composites, Master's thesis. São Carlos School of Engineering, University of São Paulo, São Carlos, São Paulo, Brazil (in portuguese)

Medeiros R, Sartorato M, Ribeiro ML, Vandepitte D, Tita V (2012) Numerical and experimental analyses about SHM metrics using piezoelectric materials. In: Proceedings of ISMA2012-USD2012. Leuven, Belgium, https://www.isma-isaac.be/ publications/proceedings.html

Medeiros R, Ribeiro ML, Sartorato M, Marinucci G, Tita V (2013) Computational simulation using PZT as sensor elements for damage detection on impacted composite cylinders. In: Proceedings of the 1st International Symposium on Solid Mechanics MecSol2013. Porto Alegre, Rio Grande do Sul, Brazil, http://www.ufrgs.br/mecsol2013/articles/MecSol2013-007.pdf

Mickens T, Schulz M, Sundaresan M, Ghoshal A, Naser A, Reichmeider R (2003) Structural health monitoring of an aircraft joint. Mech Syst Signal Process 17:285-303, doi: 10.1006/mssp.2001.1425

Military Handbook (2002) Composite materials handbook: Volume 3. Polymer matrix composites materials usage, design, and analysis. Technical report, Department of Defense - United States of America, http://www.amazon. com/Composite-Materials-Handbook-MIL-Volume-III/dp/1566768284

Monaco E, Franco F, Lecce L (2000) Experimental and numerical activities on damage detection using magnetostrictive actuators and statistical analysis. J Intell Mater Syst Struct 11:567-578, doi: 10.1106/2URV-DOHY-OQLA-HAWH

Ogisu T, Shimanuki M, Kiyoshima S, Okabe Y, Takeda N (2006) Feasibility studies on active damage detection for CFRP aircraft bonding structures. Adv Compos Mater 15:153-173, doi: 10.1163/156855106777873923

Prada MA, Toivola J, Kullaa J, Hollmén J (2012) Three-way analysis of structural health monitoring data. Neurocomputing 80:119-128, doi: 10.1016/..neucom.2011.07.030

Qing XP, Shawn J, Beard AK, Hannum R (2006a) A real-time active smart patch system for monitoring the integrity of bonded repair on an aircraft structure. Smart Mater Struct 15:N66-N73, doi: 10.1088/0964-1726/15/3/N03

Qing XP, Hian-Leng C, Beard SJ, Ooi TK, Marotta SA (2006b) Effect of adhesive on the performance of piezoelectric elements used to monitor structural health. Int J Adhes Adhes 26:622-628, doi: 10.1016/j.ijadhadh.2005.10.002

Quaegebeur N, Micheau P, Masson P, Belanger H (2011) Structural health monitoring of bonded composite joints using piezoceramics. In: Proceedings of the International Workshop Smart Materials, Structures \& NDT in Aerospace CANSMART. http://www.ndt.net/article/ndtcanada2011/papers/32_Quaegebeur.pdf

Soejima H, Ogisu T, Yoneda H, Okabe Y, Takeda N, Koshioka Y (2008) Demonstration of detectability of SHM system with FBG/PZT hybrid system in composite wing box structure. In the Proceedings of SPIE 6932, Sensors and Smart Structures Technologies for Civil, Mechanical, and Aerospace Systems 2008:69322E, doi:10.1117/12.776078

White C, Whittingham B, Li HCH, Herszberg I, Mouritz AP (2007) Vibration Based Structural Health Monitoring of Adhesively Bonded Composite Scarf Repairs [online]. In: Veidt M (ed) Proceedings of the 5th Australasian Congress on Applied Mechanics. Engineers Australia, 2007, Brisbane, Qld, pp 198-203, http://search.informit.com.au/ documentSummary; $\mathrm{dn}=219024298163172 ;$ res=IELENG

White C, Henry CH, Li BW, Herszberg I, Mouritz AP (2009) Damage detection in repairs using frequency response techniques. Compos Struct 87:175-181, doi:10.1016/j.compstruct.2008.05.010

Zagrai A, Doyle D, Arritt B (2008) Embedded nonlinear ultrasonics for structural health monitoring of satellite joints. In the Proceedings of the SPIE 6935, Health Monitoring of Structural and Biological Systems 2008:693505, doi:10.1117/12.775766

Zou Y, Tong L, Steven G (2000) Vibration-based model-dependent damage (delamination) identification and health monitoring for composite structures - a review. J Sound Vib 230:357-378, doi: 10.1006/jsvi.1999.2624

doi:10.1186/2196-4351-2-13

Cite this article as: Medeiros et al: Experimental analyses of metal-composite bonded joints: damage identification. Applied Adhesion Science 2014 2:13. 